\title{
The exploration of nanocomposition in biphasic polymer nanocolloids using a multivariate histogram method combined with cryo-VEELS
}

\author{
Ginam Kim and Deborah Meyers \\ Dow Corning Corporation, Science \&Technology, Midland, MI 48640
}

Valence Electron energy loss spectroscopy (EELS) combined with multiple least square fitting (MLLS) has been applied to enhance research for multiphase emulsion structure to provide essential understanding of where the various ingredients were partitioned within a biphasic emulsion preserved in aqueous media. ${ }^{1,2}$ For the investigation of interface between two polymer phases and surface enrichment of the PDMS lobe with the organic copolymer in the two-phase emulsion polymer particle, quantitative line profiles from the resulting compositional maps were used. However, the results obtained from a line profile method can be locally limited and the surface of the colloids still remained insufficiently characterized. To overcome this limit, we applied multivariate histograms which is a scatter diagram analysis to understand the compositional correlations of spatially aligned multiple compositional maps of the components in biphasic emulsions. Scattering diagram has been applied to composition maps generated by energy filtered TEM. $^{3}$ The 2-dimensional map of compositional ratio of PDMS to organic copolymer obtained from this method provided us better understanding of nanocomposition in nanocolloids not only in the initial stage of the synthesis but also after acrylate polymerization in poly(dimethyl siloxane) (PDMS) seed particles to high molecular weight.

The lobed nanoemulsions were synthesized by random copolymerization of acrylate monomers in the presence of seed PDMS emulsions. Ultrathin frozen specimens were prepared by a typical cryo-plunging technique. Imaging and low loss EELS spectroscopy for frozen thin specimens were done at $-165^{\circ} \mathrm{C}$ using a Gatan GIF 2002 detector combined with a $200 \mathrm{KeV}$ field emission STEM under a beam dose condition of $3000 \mathrm{e} / \mathrm{nm}^{2}$. Spatially resolved low-loss spectrum data set was generated by digitally rastering with a $10 \mathrm{~nm}$ interpixel spacing on the specimen. The multivariate histograms were created using Gatan Digital Micrograph $^{\mathrm{TM}}$ from the resulting compositional maps obtained via MLLS fitting of reference spectra collected from each pure component to the low-loss EELS spectrum data set from the specimen.

The compositional maps (Fig. 1a) of the water, PDMS, and acrylate monomer distributions in vitrified ice at the initial stage of the synthesis clearly show that the monomeric acrylates are distributed in the seed PDMS nanoparticles rather than in water. However, the bivariate histogram for the monomers and PDMS (Fig.1b) indicates that the overall concentration of monomers in the system is pretty low considering the 1:1 ratio of these two components used in the synthesis. The regions of interest (ROI) in the bivariate histogram and its resultant composite map (Fig.1c) show higher compositional ratio of monomers to PDMS at the surface (Green) than inside PDMS lobe (Red, Cyan). This result indicates surface enrichment of PDMS colloids with monomers. The results are summarized in the table of compositional ratio its correspondent color (Fig. 1d).

After the formation of biphasic emulsions, the copolymer-rich lobe in the two-phase colloid consists of almost pure organic copolymer while the PDMS-rich lobe is partly mixed with the copolymers, as shown in Figure 2a. The incomplete phase separation is also indicated by the bivariate histogram of PDMS and copolymer (Fig. 2b) showing non-bimodal pattern. The copolymer enrichment at the surface of the PDMS lobe even after copolymerization, is manifested by the ROI map and its resultant color map (Fig. 2c). As shown in the table (Fig. 2d), the compositional ratio of copolymer to PDMS is higher at the surface (Green) than inside PDMS lobe (Red). The concentration of copolymer is also higher than PDMS in the interface region (Brown). The formation of only one copolymer-rich lobe indicates that only a single nucleation event occurred for the copolymer phase. However, since we observe that prior to polymerization the acrylate monomer is not uniformly distributed throughout the PDMS seed particle, the present measurements suggests that at least some of the copolymer enrichment of the PDMS surface that we observe after polymerization may be a consequence of this pre-existing monomer distribution. 
1. Kim G.; Sousa A; Shope, M.; Meyers, D.; Libera, M. J Ameri. Chem. Soc. 128 (2006) 6571.

2. Kim G., Sousa A, Meyers D., Libera M. Microscopy and Microanalysis 14, (2008) 459.

3. Hofer F.,Grogger W., Kothleitner G., Warbichler P. Ultramicroscopy 67 (1997) 83.
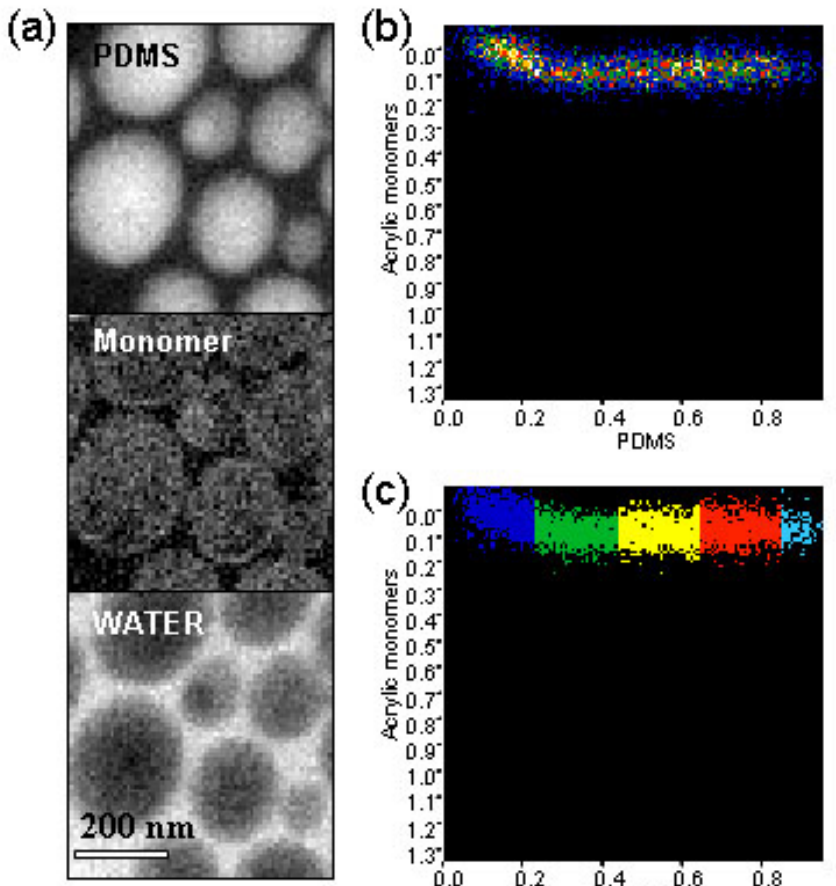

(d)
\begin{tabular}{|l|l|}
\hline $\begin{array}{l}\text { Ratio of Mo nomers } \\
\text { to PDMS }\end{array}$ & Color \\
\hline $0.33 \pm 0.14$ & Green \\
\hline $0.2 \pm 0.03$ & Rellow \\
\hline $0.13 \pm 0.03$ & Red \\
\hline $0.11 \pm 0.04$ & Cyan \\
\hline
\end{tabular}
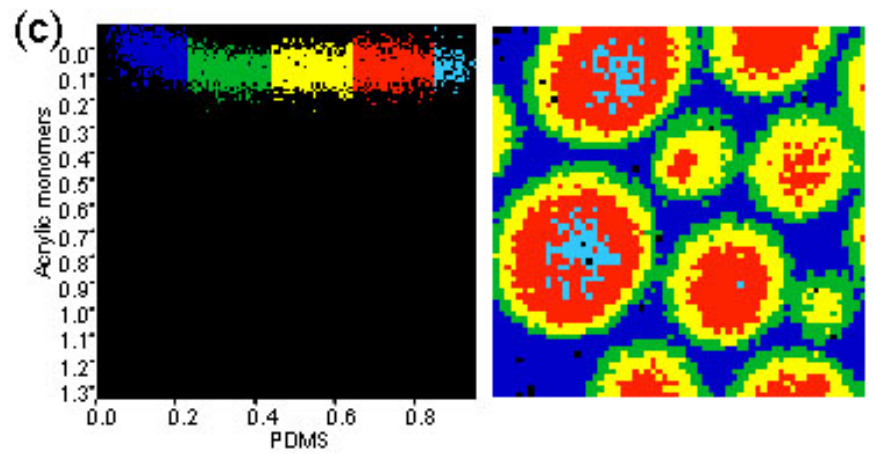

Figure.1 (a) Compositional maps of PDMS, water and monomers; (b) Bivariate compositional histogram of PDMS and monomers; (c) ROI map of bivariate histogram and its resultant composite map; (d) Table: compositional ratio of monomers to PDMS and its correspondent color in the composite map (Fig.1c)
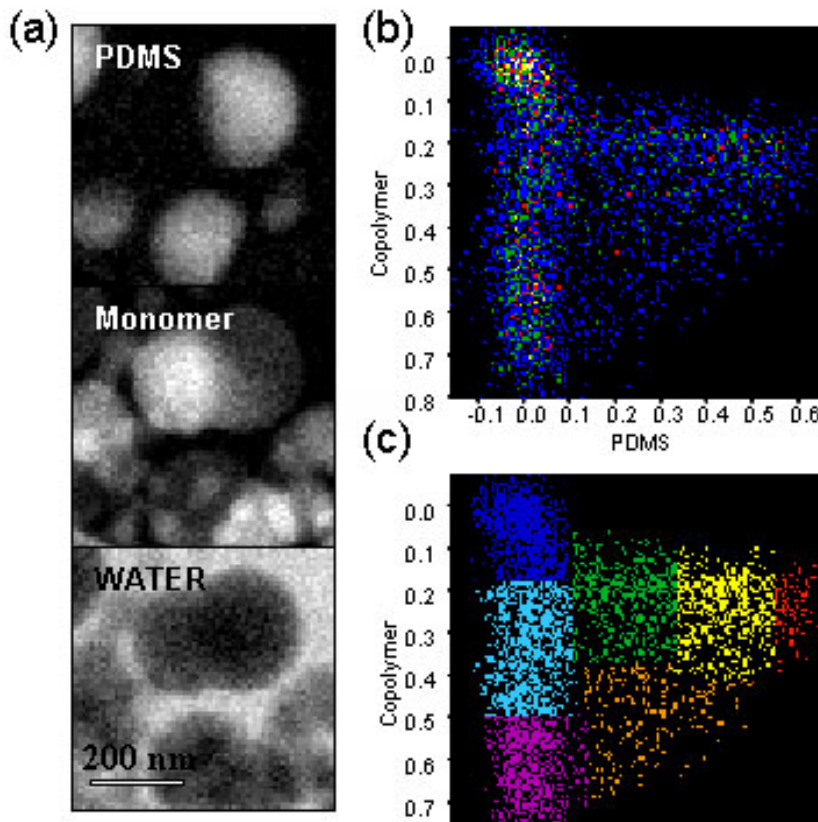

(d)
\begin{tabular}{|l|l|}
\hline $\begin{array}{l}\text { Ratio of Copolymer } \\
\text { to PDMS }\end{array}$ & Color \\
\hline $0.91 \pm 0.3$ & Green \\
\hline $052 \pm 0.21$ & Fellow \\
\hline $036 \pm 0.25$ & Red \\
\hline $1.66 \pm 0.95$ & Brown \\
\hline
\end{tabular}

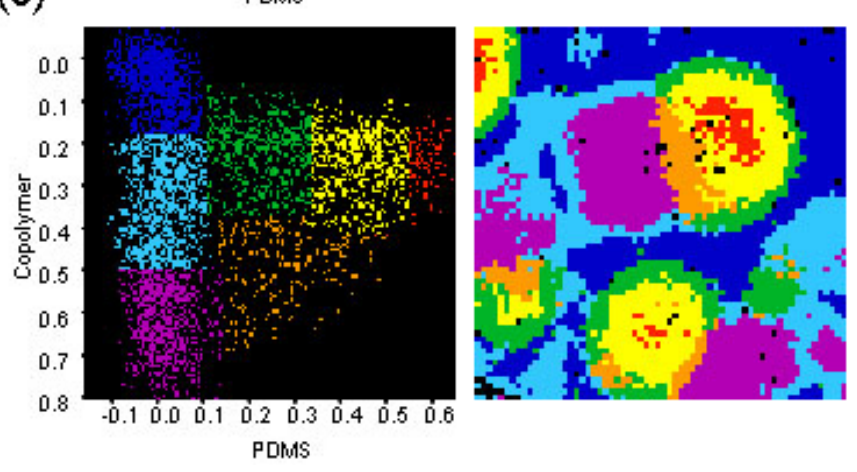

Figure.2 (a) Compositional maps of PDMS, water and copolymer, (b) Bivariate compositional histogram of PDMS and copolymers, (c) ROI map of bivariate histogram and its resultant composite map; (d) Table: compositional ratio of copolymer to PDMS and its correspondent color in the composite map (Fig.2c) 